\title{
Limited evidence on treatments for distalising upper first molars in children and adolescents
}

\author{
Abstracted from \\ Jambi S, Thiruvenkatachari B, O'Brien KD, Walsh T.
}

Orthodontic treatment for distalising upper first molars in children and adolescents. Cochrane Database Syst Rev 2013; 10: Art. No.: CD008375. DOI: 10.1002/14651858.CD008375.pub2.

Address for correspondence: Luisa Fernandez Mauleffinch, Review Group Co-ordinator, Cochrane Oral Health Group, MANDEC, School of Dentistry, University of Manchester, Higher Cambridge Street, Manchester, M15 6FH, UK. E-mail: luisa.fernandez@manchester.ac.uk

\section{Question: How effective are orthodontic appliances in distalising upper first molars in children and adolescents?}

Data sources The Cochrane Oral Health Group's Trials Register, the Cochrane Central Register of Controlled Trials (CENTRAL), Medline and Embase.

Study selection Randomised clinical trials (RCTs) involving the use of removable or fixed orthodontic appliances intended to distalise upper first molars in children and adolescents.

Data extraction and synthesis Study selection, risk of bias assessment and data extraction were carried out independently by at least two reviewers. For dichotomous outcomes the measure of treatment effect was the risk ratio; for continuous outcomes the measure of treatment effect was the mean difference. Data analysis followed Cochrane protocols.

Results Ten studies (involving 354 patients) were included in this review. Study quality was generally poor; seven studies were assessed to be at high risk of bias and three of unclear risk of bias. Meta-analyses were conducted where appropriate. Four studies (159 patients) compared a distalising appliance to an untreated control. The degree and direction of molar movement and loss of anterior anchorage varied with the type of appliance. Four studies involving (150 patients) compared a distalising appliance versus headgear. The mean molar movement for intraoral distalising appliances was $-2.20 \mathrm{~mm}$ and -1.04 $\mathrm{mm}$ for headgear.

There was a statistically significant difference in mean distal molar movement (mean difference (MD) $-1.45 \mathrm{~mm}$; 95\% confidence interval (Cl) -2.74 to -0.15 ) favouring intraoral appliances compared to headgear. However, a statistically significant difference in mean mesial upper incisor movement (MD $1.82 \mathrm{~mm} ; 95 \% \mathrm{Cl} 1.39$ to 2.24 ) and overjet (fixed-effect: MD $1.64 \mathrm{~mm} ; 95 \% \mathrm{Cl} 1.26$ to 2.02 ; two studies, unclear

This paper is based on a Cochrane Review published in the Cochrane Library 2013, issue 10 (see www.thecochranelibrary.com for information). Cochrane Reviews are regularly updated as new evidence emerges and in response to feedback, and the Cochrane Library should be consulted for the most recent version of the review. risk of bias, 70 participants analysed) favoured headgear, ie there was less loss of anterior anchorage with headgear. Owing to variation in interventions, direct comparisons of intraoral appliances from three studies (93 patients), high or unclear risk of bias was reported narratively. All appliances were reported to provide some degree of distal movement, and loss of anterior anchorage varied with the type of appliance. No included studies reported on the incidence of adverse effects (harm, injury), number of attendances or rate of non-compliance.

Conclusions It is suggested that intraoral appliances are more effective than headgear in distalising upper first molars. However, this effect is counteracted by loss of anterior anchorage, which was not found to occur with headgear when compared with intraoral distalising appliance in a small number of studies. The number of trials assessing the effects of orthodontic treatment for distilisation is low, and the current evidence is of low or very low quality.

\section{Commentary}

The area covered in this systematic review is of significant clinical interest. There is still controversy round this topic nowadays, even though we have been using some of the included appliances for more than 100 years. As expected from a Cochrane-based systematic review general principles for conducting systematic reviews were followed. I will offer some comments to generate some debate.

An area that could have improved the methodology of this systematic review would be searching Latin-American publications through Lilacs. Currently a large proportion of the manuscript submissions to the main orthodontic journals come from countries like Brazil and Turkey. Databases that targeted those countries are likely to identify potentially good studies not indexed in the regularly searched electronic databases.

A point that required clarity is the limited focus on children and adolescents (16 years-of-age was set as the upper age limit). Why not also young adults? Are anatomical differences expected? It is unlikely to be related to facial skeletal maturation, as it is known that facial growth continues until at least the twenties if not well into adulthood. Dental maturation also does not make sense. No 
indication of the effect of erupted or unerupted second or third molars was considered. Recent evidence has failed to find any significant effect of the degree of tooth eruption on the rate of first molar distalisation. It has to be said that the risk of bias among the studies considered to support this conclusion was high.

The authors state that potential drawbacks for the use of headgear are serious injuries. How frequent are these serious injuries? Are we talking about a handful of events after more than 100 years of use? Related safety developments have significantly reduced any potential of such injuries. Therefore this may be an unfair statement against a treatment alternative that when used by compliant patients, can produce remarkable changes. The authors stated that their included studies did not report adverse effects, but is it because of absence of such effects or absence of reporting?

Another area that was not considered by the included studies is the number of appointments (including emergencies) between both approaches. This has a direct impact on the efficiency of either approach. Clinically the results of the meta-analysis are eye-opening. The clinical significance of the reported changes is questionable with $1 \mathrm{~mm}$ for the headgear and $2.2 \mathrm{~mm}$ for the intraoral distalising appliance.

A large variability was identified, in addition that these analyses were based on only 354 participants. Is the extra $1.4 \mathrm{~mm}$ distalisation of such importance to consider intraoral distalising appliances a better choice? The anteriorly directed unwanted incisor movements ( $1.8 \mathrm{~mm}$, increase of overjet by $1.6 \mathrm{~mm}$ ) would further deny any advantage. Is in some cases premolar extraction not a more efficient option?

Finally, a point that I would like to raise is the inclusion of only RCTs. Due to the fact that the quality of the included RCTs was mainly poor, why not to include non-RCTs? Would they provide additional data that may be clinically useful, even considering the increased risk of bias among lower level of evidence studies? Sometimes a well-conducted non-randomised clinical trial can provide sound evidence in comparison to a poorly conducted RCT.

\section{Practice points}

- The present systematic review does not provide further clarity to this controversial area. This is not to be blamed on the author's effort, but on the reality of the limited available evidence

- Maybe an important clinical point that requires to be explored is the comparison between distalisation techniques against extraction decisions when the same treatment goal is sought.

Carlos Flores-Mir School of Dentistry, University of Alberta, Canada

Evidence-Based Dentistry (2014) 15, 23-24. doi: 10.1038/sj.ebd.6400988 